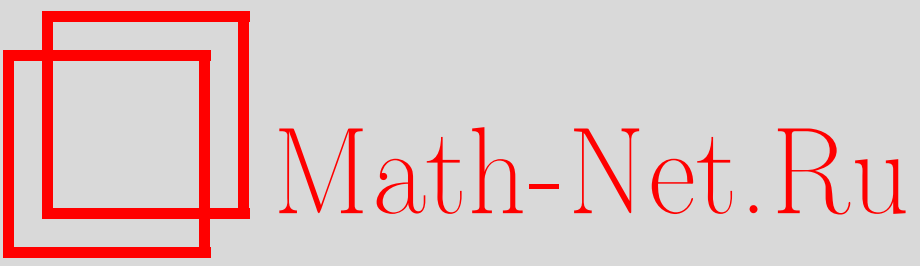

М. Б. Менский, Релятивистские квантовые измерения, эффект Унру и черные дыры, ТМФ, 1998, том 115, номер 2, 215-232

DOI: https://doi.org/10.4213/tmf868

Использование Общероссийского математического портала Math-Net.Ru подразумевает, что вы прочитали и согласны с пользовательским соглашением

http://www.mathnet.ru/rus/agreement

Параметры загрузки:

IP: 3.82 .47 .9

26 апреля 2023 г., 13:50:09 
ТЕОРЕТИЧЕСКАЯ

И МАТЕМАТИЧЕСКАЯ

ФИЗИКА

Том 115, № 2

май, 1998

(C) 1998 г

М.Б. Менский*

\section{РЕЛЯТИВИСТСКИЕ КВАНТОВЫЕ ИЗМЕРЕНИЯ, ЭФФЕКТ УНРУ И ЧЕРНЫЕ ДЫРЫ}

Показано, как применять технику ограниченных интегралов по путям или квантовых коридоров для анализа релятивистских измерений. Затем эта техника используется для того, чтобы прояснить физическую природу термических эффектов, наблюдаемых ускоренным наблюдателем в пространстве-времени Минковского (эффект Унру) и далеким наблюдателем в поле черной дыры (эффект Хокинга). Физическая природа "термической атмосферы" вокруг наблюдателя анализируеся в трех случаях: а) эфффект Унру, б) вечная (шварцшильдовская) черная дыра и в) черная дыра, образующаяся в процессе коллапса. Показано, что термические частицы реальны только в случае "в". В случае "б" они неотличимы от реальных частиц, но не уносят массу черной дыры до тех пор, пока некоторые из этих частиц не поглощаются далеким наблюдателем. В случае "а" термические частицы виртуальны.

\section{1. ВВЕДЕНИЕ}

Нерелятивистская квантовая теория измерений сушественно использует постулат фон Неймана и не может применяться к релятивистским системам из-за нарушения причинности при мгновенной редукции состояния измеряемой системы. Эта проблема рассматривалась многими авторами (см., например, [1-4]), но соглашения о том, как корректно описывать релятивистские квантовые измерения, достигнуто не было. Общий вывод из этой дискуссии состоит в том, что в релятивистском случае нельзя пренебрегать длительностью квантового измерения и размерами пространственной области, в которой оно проводится. Релятивистские квантовые измерения должны рассматриваться как непрерывные в пространстве и во времени.

Подход, основанный на ограниченных интегралах по путям (ОИП), был успешно применен ранее как к релятивистским, так и к нерелятивистским схемам измерения [5-7]. В частности, в работе [7] этот подход использовался для описания измерения положения релятивистской частицы.

\footnotetext{
* Физический институт им. П. Н. Лебедева РАН, Москва, Россия
} 
В данной работе мы разовьем подход ОИП так, чтобы его можно было применять к широкому кругу квантовых измерений над элементарными частицами. Затем с помощью этой техники будут сделаны некоторые качественные выводы относительно эффекта Унру и эффекта Хокинга.

Идея подхода ОИП была сформулирована Фейнманом [8] для описания непрерывных (дляшихся во времени) нерелятивистских квантовых измерений и разработана технически и распространена на новые области в работах $[5,6,9]$ (см. также [10]). Важное преимушество данного подхода - это его обшность и независимость от каких бы то ни было конкретных моделей измерения.

Центральной идеей подхода ОИП является то, что эволюция системы, подвергающейся непрерывному измерению, должна описываться интегралом по путям, в котором область интегрирования ограничена на множество путей, совместимых с результатом измерения. Следовательно, вместо обычного фейнмановского интеграла по всем путям возникает интеграл по коридору путей. Такой коридор путей может быть назван квантовыцм коридором по аналогии с близким (но не тождественным) понятием квантовой траектории, введенной Кармайклом [11]. Квантовые коридоры играют важную роль в интерпретации непрерывных квантовых измерений. Определенный набор квантовых коридоров определяет непрерывное измерение (аналогично тому, как набор проекторов определяет фон-неймановское измерение). Альтернативные квантовые коридоры из этого набора соответствуют альтернативным результатам, которые могут возникнуть при рассматриваемом измерении.

В данной работе мы сформулируем основные пункты метода квантовых коридоров для релятивистских элементарных частиц. Затем понятие квантового коридора будет использовано для анализа некоторых концептуальных проблем, возникаюших в связи с эффектами Унру и Хокинга. В процессе анализа мы проясним физическую природу “термической атмосферы”, наблюдаемой ускоренным наблюдателем в пространстве-времени Минковского или наблюдателем, движушимся вдали от черной дыры (ЧД). Более конкретно мы ответим на следующие вопросы:

1. Можно ли в ходе наблюдения термических эффектов разделить вклады отдельных частиц, составляющих термическую атмосферу?

2. Являются ли эти частицы реальными, т.е. можно ли каждую из них наблюдать так, чтобы сам факт ее существования был независим от измерения?

Мы увидим, что ответы на эти вопросы различны для ускоренного наблюдателя, для наблюдателя в поле "вечной" ЧД (описываемой метрикой Крускала) и для наблюдателя в поле ЧД, возникающей в процессе коллапса. Некоторые из выводов, к которым мы придем, являются, конечно, известными, в частности из важной работы Унру и Вальда [12]. Однако некоторые из них, особенно различие между вечной ЧД и такой ЧД, которая возникла в результате коллапса, по-видимому, не были сформулированы достаточно ясно. Во всяком случае, эти вопросы получают новое освещение в подходе ОИП. 


\section{2. РЕЛЯТИВИСТСКИЕ ИНТЕГРАЛЫ ПО ПУТЯМ}

Причинный пропагатор (амплитуда распространения) для релятивистской частицы можно представить в форме интеграла по путям, если, кроме четырех пространственно-временных координат, ввести, следуя Штюкельбергу [13], пятый параметр $\tau$, который называется собственным временем или историческим временем.

Рассмотрим для простоты скалярную частицу массы $m$. Ее причинныц й попагатор равен интегралу по собственному времени ${ }^{1)}$

$$
K\left(x^{\prime \prime}, x^{\prime}\right)=\int_{0}^{\infty} d \tau \exp \left(-i\left(m^{2}-i \epsilon\right) \tau\right) K_{\tau}\left(x^{\prime \prime}, x^{\prime}\right)
$$

от вспомогательного пропагатора, зависящего от собственного времени. Последний, в свою очередь, может быть записан в форме интеграла по путям

$$
K_{\tau}\left(x^{\prime \prime}, x^{\prime}\right)=\int_{x^{\prime \prime} \leftarrow x^{\prime}} d[x]_{\tau} \exp \left(-\frac{i}{4} \int_{0}^{\tau}(\dot{x}, \dot{x}) d \tau\right) .
$$

Здесь $(\cdot, \cdot)$ обозначает лоренщеву свертку, а путь $[x]_{\tau}$ между точками $x^{\prime}$ и $x^{\prime \prime}$ пространства-времени Минковского параметризован интервалом собственного времени $[0, \tau]^{2)}$.

Как следствие этих определений вспомогательный пропагатор, зависяший от собственного времени, удовлетворяет "релятивистскому уравнению Шредингера"

$$
\frac{d}{d \tau} K_{\tau}\left(x^{\prime \prime}, x^{\prime}\right)=-i \square K_{\tau}\left(x^{\prime \prime}, x^{\prime}\right),
$$

а причинный пропагатор $K\left(x^{\prime \prime}, x^{\prime}\right)$ является функцией Грина уравнения Клейна-Гордона

$$
\left(\square+m^{2}\right) K\left(x^{\prime \prime}, x^{\prime}\right)=-i \delta\left(x^{\prime \prime}, x^{\prime}\right) .
$$

Будучи функцией Грина, пропагатор $K\left(x^{\prime \prime}, x^{\prime}\right)$ удовлетворяет важному соотношению

$$
i \int_{\mathcal{S}} \sigma^{\mu} K\left(x^{\prime \prime}, x\right) \overleftrightarrow{\partial}_{\mu} K\left(x, x^{\prime}\right)=K\left(x^{\prime \prime}, x^{\prime}\right)
$$

в котором $\mathcal{S}$ обозначает замкнутую гиперповерхность такую, что точка $x^{\prime}$ находится внутри, а $x^{\prime \prime}$ - вне ее ${ }^{3)}, \sigma^{\mu}$ - элемент площади гиперповерхности и $\stackrel{\leftrightarrow}{\partial}_{\mu}$ определено следуюшим образом:

$$
f(x) \overleftrightarrow{\partial}_{\mu} g(x)=f(x) \frac{\stackrel{\leftrightarrow}{\partial}}{\partial x^{\mu}} g(x)=f(x) \frac{\partial g(x)}{\partial x^{\mu}}-\frac{\partial f(x)}{\partial x^{\mu}} g(x)
$$

\footnotetext{
1) В данной работе мы используем естественные единицы измерения $\hbar=c=1$.

2) Заметим, что определенное таким образом "собственное время" не совпадает с тем, которое называется собственным временем в классической физике (собственным временем наблюдателя на данной траектории). Термин "историческое время" кажется более подходящим. Однако термин "собственное время" используется в данном контексте чаще.

3) Аналогичное соотношение, но с противоположным знаком в правой части, справедливо для гиперповерхности $\mathcal{S}$, по отношению к которой $x^{\prime \prime}$ находится внутри, а $x^{\prime}$ - снаружи.
} 
Эти свойства пропагатора могут быть обобщены на случай произвольного внешнего электромагнитного или гравитационного поля. Все производные должны в этом случае быть ковариантными, и интеграл по путям (2) должен быть определен ковариантным образом [14].

Для анализа непрерывных измерений релятивистских частиц в рамках подхода ОИП мы должны привлечь интегралы по путям типа (1), (2), но с интегрированием, ограниченным семейством путей, совместимых с соответствуюшим результатом измерения.

\section{3. ИЗМЕРЕНИЯ ЧАСТИШ}

Рассмотрим кратко основные черты релятивистского метода ОИП начиная с простого случая измерения положения частицы.

3.1. Измерение положения. Измерение положения частищы в момент времени $x^{0}=t$, приводяшее к результату измерения $\mathbf{x}=\mathbf{a}$, можно описать [7] интегралом по путям, пересекаюшим пространственноподобную поверхность $\mathcal{S}=\left\{x \mid x^{0}=t=\right.$ const $\}$ в узкой области вокруг точки $a=(t, \mathbf{a})$ (см. рис. 1a). Мы будем говорить, что пути проходят через ворота в поверхности $\mathcal{S}$, причем положение ворот а соответствует результату измерения, а ширина $\Delta a$ определяет то, с каким разрешением производится измерение (какова ошибка измерения).

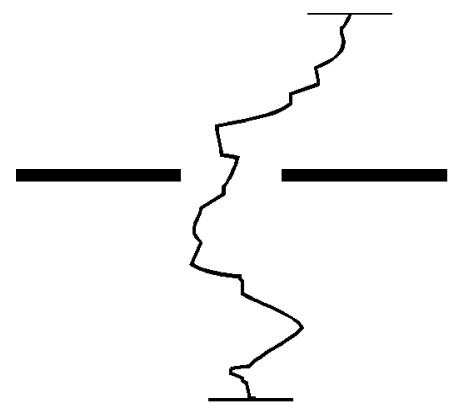

$\mathrm{a}$

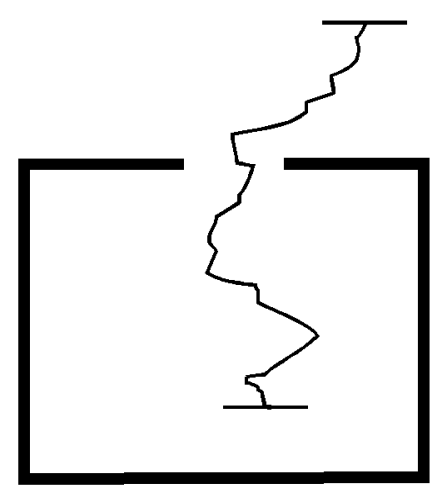

б

Рис. 1. Измерение положения релятивистской частицы, представленное путями, проходящими через ворота в гиперповерхности постоянного времени (а) или в замкнутой гиперповерхности (б). Короткие горизонтальные линии - пространственноподобные гиперповерхности, на которых регистрируются входяшие и выходящие частицы.

ЗАмЕчАнИЕ. На самом деле поверхность $\mathcal{S}$ должна быть замкнутой, как показано на рис. 1б, в соответствии с соотношением (5). В случае нулевого внешнего поля интеграл по прошлой пространственноподобной гиперповерхности, входяшей в состав замкнутой 
гиперповерхности $\mathcal{S}$, а также интеграл по времениподобной боковой гиперповерхности равны нулю, если эти гиперповерхности расположены достаточно далеко. Следовательно, поверхность $\mathcal{S}$ в этом специальном случае может быть выбрана в виде временного сечения $\left\{x \mid x^{0}=t=\mathrm{const}\right\}$. В данной работе мы рассмотрим общую ситуацию, поэтому замкнутые поверхности с воротами будут играть главную роль.

Таким образом, результат измерения, равный $a \in \mathcal{S}$, полученный при измерении с конечным разрешением, можно описать узкой областью (воротами) $G(a)$ на поверхности $\mathcal{S}$ вокруг точки $a \in \mathcal{S}$. Соответствуюшая амплитуда $K^{G(a)}\left(x^{\prime \prime}, x^{\prime}\right)$ должна быть определена как интеграл по путям, идушим из точки $x^{\prime}$ в точку $x^{\prime \prime}$ через ворота $G(a)$. С физической точки зрения эта амплитуда описывает эволюцию частицы, подвергающейся измерению при условии, что измерение дает результат $a$.

С математической точки зрения такой ОИП представляет собой произведение двух интегралов, один - интеграл по путям, идушим из точки $x^{\prime}$ к воротам $G(a)$, другой интеграл по путям, идушим от $G(a)$ к точке $x^{\prime \prime}$. Каждый из двух последних интегралов близок к полному пропагатору между соответствуюшими точками (хотя и не равен ему). Это является основанием, для того чтобы определить амплитуду $K^{G(a)}\left(x^{\prime \prime}, x^{\prime}\right)$ непосредственно через эти пропагаторы [7]:

$$
K^{G(a)}\left(x^{\prime \prime}, x^{\prime}\right)=\int_{b \in G(a)} \sigma^{\mu}(b) K_{\mu}^{(b)}\left(x^{\prime \prime}, x^{\prime}\right)
$$

где

$$
K_{\mu}^{(b)}\left(x^{\prime \prime}, x^{\prime}\right)=i K\left(x^{\prime \prime}, b\right) \overleftrightarrow{\partial}_{\mu}(b) K\left(b, x^{\prime}\right)
$$

Таким образом, формула (6) определяет ОИП, задаваемый коридором, представленным на рис. 1. Этот коридор есть (замкнутая) гиперповерхность с воротами. В следующих разделах мы рассмотрим различные обобшения такого коридора.

Амплитуды (7) введены для частицы, которая находится в пространственно-временной точке $x^{\prime}$ перед измерением и в точке $x^{\prime \prime}$ посленего. Реальная ситуация соответствует начальному и конечному состояниям, заданным волновыми функциями в соответствующие моменты времени $t^{\prime}, t^{\prime \prime}$ (эти моменты представлены на рис. 1 короткими горизонтальными линиями). В этом случае следует умножить амплитуду измерения (7) на соответствуюшие волновые функции и проинтегрировать по временным сечениям $t^{\prime}$ и $t^{\prime \prime}$ :

$$
K_{\kappa}^{(b)}\left(\psi^{\prime \prime}, \psi^{\prime}\right)=-\int \sigma^{\mu}\left(x^{\prime \prime}\right) \sigma^{\nu}\left(x^{\prime}\right) \overline{\psi^{\prime \prime}\left(x^{\prime \prime}\right)} \overleftrightarrow{\partial}_{\mu}\left(x^{\prime \prime}\right) K_{\kappa}^{(b)}\left(x^{\prime \prime}, x^{\prime}\right) \overleftrightarrow{\partial}_{\nu}\left(x^{\prime}\right) \psi^{\prime}\left(x^{\prime}\right)
$$

(черта над буквой обозначает комплексное сопряжение).

Можно показать, что соотношение (5) (соответствующее в нерелятивистском случае сохранению вероятностей или унитарности оператора эволюции) ведет к "обобщенной 
унитарности" амплитуды измерения, если только размер области $G(a)$ больше, чем комптоновская длина волны $\lambda_{C}=1 / m$ частицы, подвергающейся измерению (см. [7]). Физическая причина этого состоит в следуюшем. Локализация частицы в области размером $\Delta a$ требует энергии порядка $1 / \Delta a$, что может привести к рождению пар, если $\Delta a<\lambda_{C}$. Такое рождение пар, не вызванное внешней причиной, а индуцированное самим измерением, искажает картину событий, является "измерительным шумом". Условие $\Delta a>\lambda_{C}$ - это гарантия того, что измерительный шум пренебрежимо мал, а наблюдаемая частица реальна.

Условие $\Delta a>\lambda_{C}$ имеет смысл лишь для массивной частицы. Однако для безмассовой частишы можно применять вместо него более обшее условие $\Delta a>\lambda$, в котором $\lambda=1 / p$ - "типичная" длина волны, характерная для условий, в которых происходит измерение. Она определяется через “типичный" импульс $p$. Это условие гарантирует, что локализация частицы в области размерами $\Delta a$ не приводит к рождению пар с импульсами того же порядка, что и импульс измеряемой частишы. Измерительньй шум в этом случае мал в той области импульсов (длин волн), которая нас интересует. Наблюдаемая в этой области частица может интерпретироваться как реальная.

3.2. Другие релятивистские измерения. Многие измерения (реальные или мысленные эксперименты) элементарных частищ можно охарактеризовать в рамках подхода ОИП коридорами путей (квантовыми коридорами), т.е. замкнутыми гиперповерхностями, возможно, с некоторым числом ворот в них. Примеры даны на рис. 2. На рис. 2а и 2в представлены две альтернативные схемы наблюдения рождения пары, а на рис. $2 б$ и $2 г-$ две схемы наблюдения причинного зигзага.
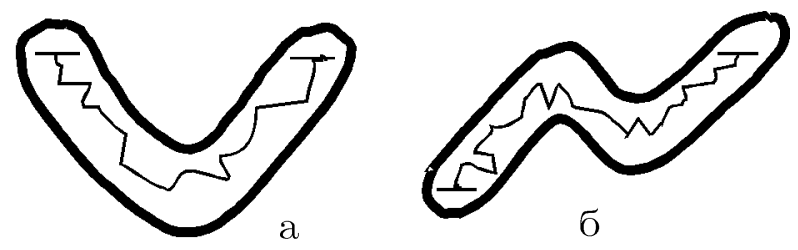

б

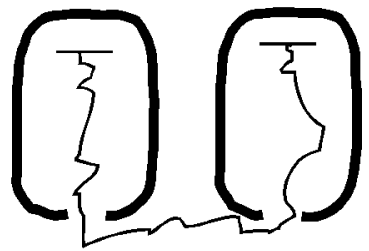

$\mathrm{B}$

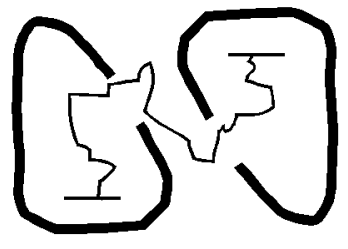

$\Gamma$

Рис. 2. Релятивистские измерения, представленные коридорами путей (квантовыми коридорами) в пространстве-времени: рождение пар (а, в) и причинный зигзаг (б, г).

Процесс измерения всегда предполагает возможность альтернативных результатов 
измерения. Если измерение описывается квантовым коридором, различные альтернативы соответствуют различным коридорам. Так, V-образный коридор на рис. 2a - это лишь один из многих альтернативных $\mathrm{V}$-образных коридоров с различными положениями вершины. Аналогично, коридор, изображенный на рис. 2б, - это один из коридоров, описываюших распространение с одного временного сечения на другое временное сечение, при котором траектория распространяюшейся частицы наблюдается (измеряется) с конечной точностью. Для реализации этих обоих типов измерения необходима среда, состоящая из объектов (например, фотонов), слабо взаимодействуюших с измеряемой частицей и таким образом локализуюших ее с конечным разрешением в пространстве и во времени.

Другой тип измерения соответствует коридорам с воротами, изображенным на рис. 2в и 2г. В этом случае (так же как и в случае измерения положения, см. рис. 1) замкнутая поверхность фиксирована, а альтернативные результаты измерения соответствуют различным положениям ворот ${ }^{4)}$. Эта формальная схема описывает наблюдение, организованное на заданной замкнутой гиперповерхности. Ширина ворот представляет разрешение, с которым совершается наблюдение. Для такого измерения требуется сеть объектов, которые активизируются в заданные моменты времени. Нет необходимости конкретизировать детали реализации измерения, т.к. метод квантовых коридоров (подход ОИП) не зависит от конкретной измерительной процедуры, а лишш от типа информации об измеряемой системе, которую эта процедура предоставляет.

Сушественно, насколько широким является коридор или ворота. Чтобы прояснить этот вопрос, следовало бы вычислить ОИП в ситуации, когда нет никаких внешних полей, которые могли бы вызвать нетривиальные процессы (например, рождение пар или причинный зигзаг). Можно ожидать, что в этой ситуации все интегралы, соответствуюшие рис. 2 , должны иметь пренебрежимо малые величины, и показать, что это имеет место, если коридоры и ворота шире, чем комптоновская длина волны измеряемой частишы, $\Delta a \gg \lambda_{C}$, или в более обшем случае шире, чем типичная длина волны измеряемой частицы, $\Delta a \gg \lambda$.

Если ширина коридора или ворот меньше (или порядка) комптоновской длины $\lambda_{C}$, то результат вычисления ОИП может быть ненулевым даже для нулевых внешних полей. Физическая причина этого состоит в том, что локализация частицы в области, имеющей размер меньше, чем $\lambda_{C}$, требует внесения энергии, большей, чем собственная энергия частищы. Эта энергия может приводить к рождению пар. В этом случае пары рождаются из-за слишком детального наблюдения того, что происходит. Рождение пар является тогда эффектом измерения, а не следствием какой-либо внешней причины. Если $\Delta a \gg \lambda_{C}$, рождения пар под влиянием измерения не происходит. Наблюдаемые частицы являются в этом случае реальными. Более слабое условие $\Delta a \gg \lambda$, где $\lambda$ -

\footnotetext{
4) Ниже мы рассмотрим такие схемы измерения, в которых число ворот также может быть различным для разных результатов измерения.
} 
типичная длина волны измеряемой частицы, гарантирует, что не могут рождаться частицы с импульсами того порядка, который нас интересует. В этом случае наблюдаемая частица также является реальной в той мере, в какой мы не интересуемся импульсами, много меньшими, чем $1 / \Delta a$.

Рассматривая измерения частиц в ненулевых полях, мы должны, следовательно, выбирать достаточно широкие коридоры и ворота $\left(\Delta a>\lambda_{C}\right.$ или $\left.\Delta a>\lambda\right)$, чтобы избежать слишком сильного обратного влияния измерения на измеряемую частицу. Если, напротив, цель исследования состоит в том, чтобы изучить влияние измерения, то ширина коридора или ворот может быть меньше, чем длина волны частицы.

\section{4. ЭФФЕКТ УНРУ}

Как было показано в работе Унру [15], ускоренньй наблюдатель в пространстве-времени Минковского увидит вакуум как тепловую баню с температурой, пропорциональ-

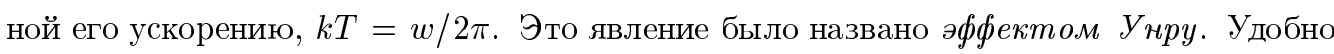
анализировать эффект Унру в координатах Риндлера $(\eta, \xi)$, которые связаны с координатами Минковского $\left(x^{0}, x^{1}\right)$ преобразованием

$$
x^{0}=\frac{1}{w} e^{w \xi} \operatorname{sh} w \eta, \quad x^{1}=\frac{1}{w} e^{w \xi} \operatorname{ch} w \eta .
$$

Траектория ускоренного наблюдателя имеет в координатах Риндлера простой вид $\xi=0$, а риндлеровское время $\eta$ - это собственное время (в классическом смысле этого слова) на этой траектории. Поверхности $x^{1}= \pm x^{0}$ являются горизонтами событий ускоренного наблюдателя. Это значит, что с ним могут быть причинно связаны только те события, которые лежат в том же самом квадранте относительно горизонтов, что и сам наблюдатель. Этот квадрант иногда называется "риндлеровским углом".

Причинный пропагатор (безмассовой) частицы в пространстве-времени Минковского между двумя точками на траектории ускоренного наблюдателя с точностью до числового фактора равен [16]

$$
\frac{w^{2} / 4}{\operatorname{sh}^{2} \frac{1}{2} w\left(\eta^{\prime}-\eta^{\prime \prime}\right)}=\sum_{n=-\infty}^{\infty} \frac{1}{\left[\left(\eta^{\prime}-\eta^{\prime \prime}\right)+i \beta n\right]^{2}},
$$

где $\beta=(k T)^{-1}$. В представлении энергии-импульса

$$
\frac{w^{2} / 4}{\operatorname{sh}^{2} \frac{1}{2} w\left(\eta^{\prime}-\eta^{\prime \prime}\right)}=-\frac{1}{(2 \pi)^{2}} \int d E d \mathbf{p} e^{i E\left(\eta^{\prime}-\eta^{\prime \prime}\right)} D_{\beta}(E, \mathbf{p})
$$

пропагатор имеет вид

$$
D_{\beta}(E, \mathbf{p})=\frac{i}{E^{2}-\mathbf{p}^{2}+i \epsilon}+\frac{2 \pi \delta\left(E^{2}-\mathbf{p}^{2}\right)}{e^{\beta|E|}-1}
$$


причем первый член соответствует члену $n=0$ в формуле (10).

Это означает, что обычный пропагатор в пространстве-времени Минковского имеет вид термического пропагатора по отношению к риндлеровскому времени (собственному времени ускоренного наблюдателя). Формально это ведет к заключению, что ускоренный наблюдатель увидит вместо вакуума тепловую баню. В разложении (10) члены с $n \neq 0$ ответственны за термические эффекты.

Как показано в работах Труста и Ван Дама [16], в представлении пропагатора в виде интеграла по путям член с данным.м $n$ представлен путями, имеющими порядок $n$ относительно начала отсчета плоскости $\left(x^{0}, x^{1}\right)$. Это значит, что только те пути, которые обходят вокруг начала отсчета точно $n$ раз, дают вклад в $n$-й член разложения (10) (рис. 3). Следовательно, термические эффекты представлены топологически нетривиальными путями, имеюшими порядок $n \neq 0$.
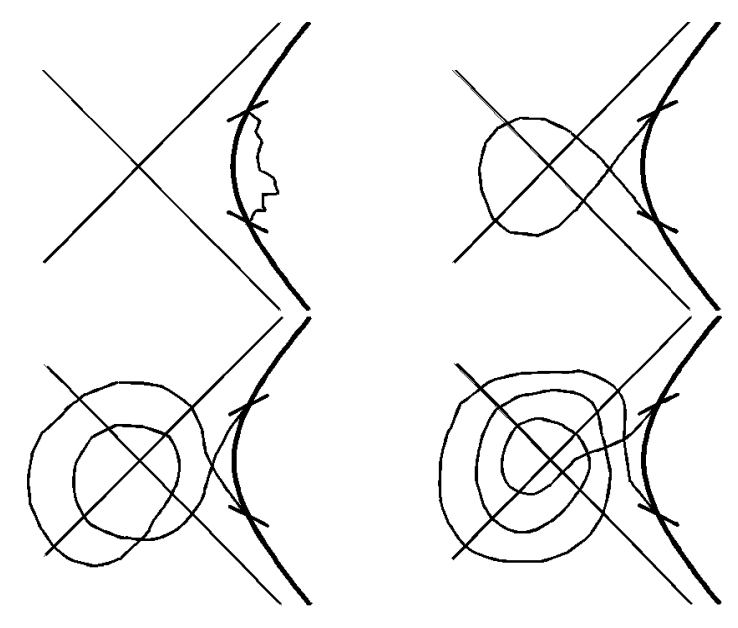

Рис. 3. Пути, имеющие различный порядок $n$ относительно начала отсчета риндлеровской плоскости. Толстая линия - траектория ускоренного наблюдателя, тонкие прямые линии - его горизонты событий.

Применим подход ОИП для анализа эффекта Унру. Рассмотрим сначала измерительную схему, которая не индуцирует рождения пар. Согласно сказанному в конце пункта 3.2 , если мы хотим организовать наблюдение таким образом, чтобы оно само по себе не индуцировало рождения пар, то квантовые коридоры, описываюшие соответствуюшую измерительную схему, должны иметь размер больше, чем длина волны интересующих нас частиц. В данном случае нас интересуют частицы, образуюшие тепловую баню, и следует позаботиться, чтобы такие частицы не могли рождаться вследствие измерительной процедуры. Типичная энергия частиц в тепловой бане есть $k T$, так что их типичная длина волны равна $1 / k T$. Коридоры и ворота, описываюшие измерение, должны иметь ширину, бо́льшую чем $\lambda=1 / k T$. 
Можно показать, что любая точка на траектории ускоренного наблюдателя отделена расстоянием порядка $\lambda$ от соответствующей точки на "траектории антинаблюдателя", получаемой отражением относительно начала отсчета, $x^{0} \rightarrow-x^{0}, x^{1} \rightarrow-x^{1}$. Следовательно, среди всех альтернативных широких (по сравнению с длиной волны) коридоров мы должны рассмотреть и такие коридоры, которые включают вместе с любой частью траектории наблюдателя также соответствуюшую часть траектории "антинаблюдателя", а также всю область, лежашую между этими траекториями. Очевидно, что начало отсчета риндлеровской плоскости, а следовательно, и все топологически нетривиальные пути, ответственные за термические эффекты, окажутся внутри такого коридора (рис. 4а). Индивидуальные частицы, составляюшие "тепловую атмосферу" ускоренного наблюдателя, не могут быть разделены с помошью измерения этого типа. Термические эффекты интерпретируются в этом случае как "вакуумные флюктуации в вакууме Минковского" [12].

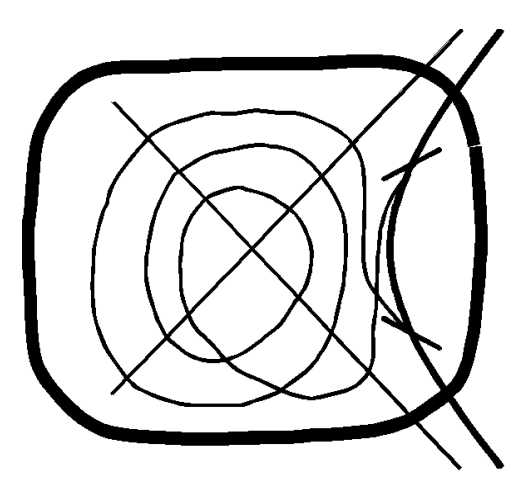

a

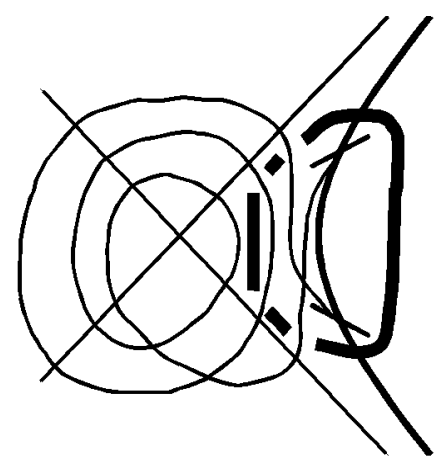

б

Рис. 4. Наблюдение эффекта Унру: а - “термический эффект" как вакуумные флюктуации (широкий коридор); б - наблюдение отдельных термических частиц (узкий коридор).

Рассмотрим теперь другой тип измерения, для которого все альтернативные коридоры ограничены горизонтами событий ускоренного наблюдателя (находятся внутри "риндлеровского угла"). В этом случае коридоры являются узкими (в сравнении с длиной волны). В таком измерении рождение пар как эффект измерения не может быть исключено. Проанализируем этот тип измерений.

Узкие коридоры можно выбрать таким образом, чтобы охарактеризовать термические эффекты более детально. Например, квантовый коридор, представленный на рис. 4б, дает амплитуду для распространения частицы с одновременным созданием не менее двух термических частиц $(n \geq 2)$, одна из которых свободно распространяется через область измерения. Из рис. 4 видно, что термический эффект будет интерпре- 
тирован соответствуюшим наблюдателем как наличие частиц, приходяших со стороны прошлого горизонта и уходящих за будуший горизонт. Амплитуды, соответствуюшие коридорам этого типа, можно вычислить и в принципе сравнить с экспериментальными данными. Однако коридор в этом случае будет узким (по сравнению с длинами волн термических частиц). Это значит, что в соответствуюших экспериментах влияние измерительной процедуры не является пренебрежимо малым. Наблюдаемые частицы не могут, следовательно, интерпретироваться как реальные частицы, сушествуюшие независимо от измерения.

Можно рассмотреть интегралы по путям, описываюшие поглошение частишы ускоренным наблюдателем (рис. 5). Поглошение "риндлеровской частицы" сопровождается в этом случае рождением еше одной частицы, которая может быть поглощена инерциальным наблюдателем [12]. В этом случае сушествует широкий коридор (с широкими воротами), как показано на рис. 5а. Он включает все термические члены (все возможные числа $n$ ). Поглошение "риндлеровской частищы" и сопровождаюшее его излучение “частицы Минковского” являются реальными (не виртуальными) процессами, но вклады различных $n$ в эти процессы нельзя разделить экспериментально. Более детальное наблюдение, разделяюшее эти вклады, описьвается узкими квантовыми коридорами (рис. 5б). Однако влияние измерительной процедуры не является в этом случае пренебрежимым. Обнаруживая термические частицы, наблюдатель не может интерпретировать их как реальные частищы, сушествуюшие независимо от наблюдения.

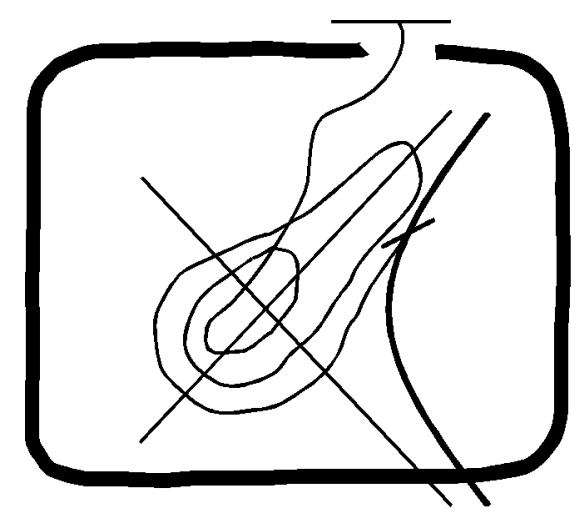

a

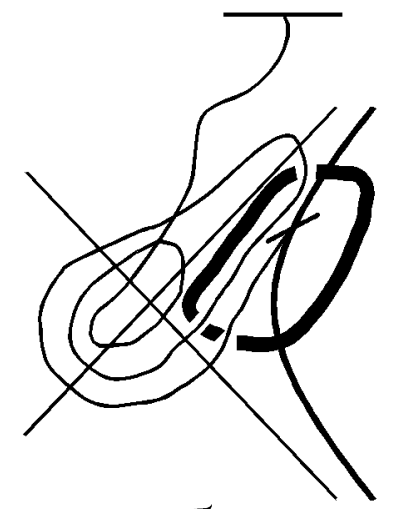

б

Рис. 5. Поглощение частицы ускоренным наблюдателем: a - широкий коридор, описывающий наблюдение двух реальных процессов (поглощения "риндлеровской частицы" и рождения "частицы Минковского"); б - узкий коридор, разделяющий вклады отдельных термических частиц.

Выводы для эффекта Унру:

3 Теоретическая и математическая физика, т. 115, № 2, 1998 г. 
А. "Тепловая атмосфера" ускоренного наблюдателя состоит из виртуальных (а не реальных) частии, которые являются лишь частями длинной петли, представляющей вакуумные флюктуации (рис. 4а).

Б. Наблюдение, организованное в узкой области (по сравнению с длинами волн термических частии), может привести к “обнаружению” отдельных термических частиц, однако при этом нельзя исключить обратного влияния измерительной прочедурь (рис. 4б).

В. Если термическая частица поглощается ускоренным наблюдателем, то петля разрывается и соответствующая античастица становится реальной и может быть наблюдена инерциальным наблюдателем как реальная частица (puc.5a).

\section{5. ЧЕРНЫЕ ДЫРЫ}

Теоретически могут сушествовать два качественно различных типа черных дыр (ЧД) (см. рис.6) ${ }^{5)}:$ вечная ЧД и ЧД, формирующаяся в процессе коллапса обычной материи (например, звезды). Вечная ЧД (если она имеет нулевые угловой момент и заряд) описывается метрикой Крускала и имеет два горизонта событий (будуший и прошлый) и две сингулярности (будушую и прошлую) (см. рис. 6а). В отличие от этого ЧД, формирующаяся в процессе коллапса, имеет лишь один (будущий) горизонт и лишь одну (будушую) сингулярность. Для обоих типов ЧД на рис. 6 изображена траектория наблюдателя, движушегося на постоянном расстоянии от ЧД.

Хокинг показал [18], что наблюдатель, движущийся далеко от ЧД, увидит тепловую баню, имеюшую температуру, обратно пропорциональную массе ЧД: $k T=1 / 8 \pi G M$, где $G$ - гравитационная постоянная. Однако природа этого термического эффекта не совсем ясна до сих пор $[19,20]$. Мы используем подход ОИП, чтобы проанализировать этот вопрос.

5.1. Вечная черная дыра. Как было показано в работе Труста и Ван Дама [16], термические эффекты в поле вечной ЧД в полной аналогии с эффектом Унру связаны с топологически нетривиальными путями на этот раз относительно начала отсчета координат Крускала (это точка, в которой пересекаются горизонты). Так же как в плоскости Риндлера, порядок (топологическое число) $n$ данного пути относительно начала отсчета плоскости Крускала равен числу термических частиц. Мы проанализируем эти пути с помошью различных квантовых коридоров (рис. 7), тем самым выясняя, как проявляются термические эффекты в измерениях того или иного типа.

Несмотря на глубокие аналогии одна черта сушественно отличает эффект Хокинга от эффекта Унру. В случае эффекта Унру температура стремится к нулю, когда ускоре-

\footnotetext{
5) Мы предполагаем, что читатель знаком с основными сведениями о ЧД, которые можно найти, например, в [17].
} 


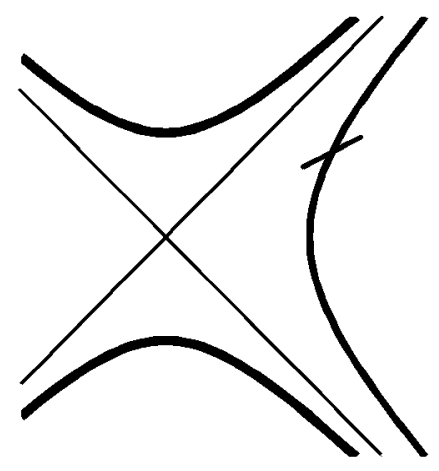

a

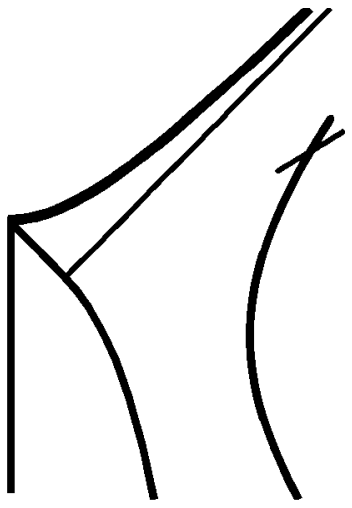

б

Рис. 6. Два типа черных дыр: а - вечная (щварцшильдовская) ЧД, имеющая будущий и прошлый горизонты событий (тонкие прямые линии) и будущую и прошлую сингулярности (толстые линии в нижней и в верхней частях диаграммы); б - ЧД, формирующаяся в процессе коллапса, имеющая только будущую сингулярность и только будущий горизонт. Толстая линия справа - траектория далекого наблюдателя.

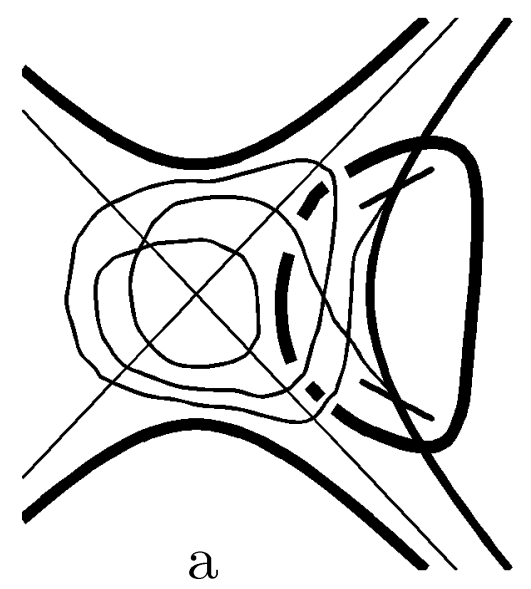

$a$

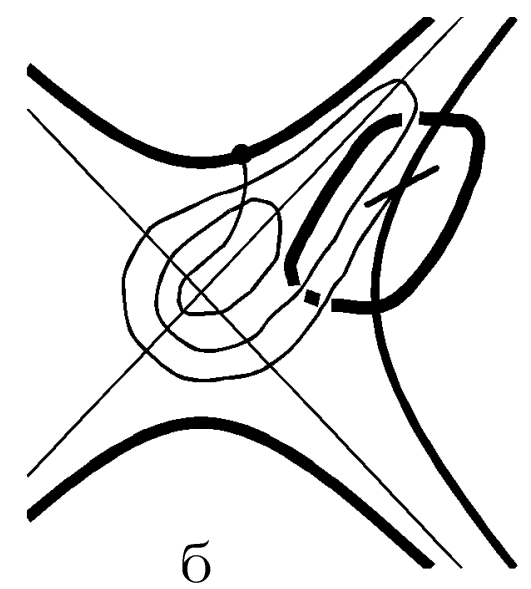

б

Рис. 7. Наблюдения в поле вечной Чд: а - наблюдение, организованное в широкой области; б - поглощение частицы, наблюдаемое в широкой области.

ние $w$ уменьшается, т.е. для наблюдателя, далекого от начала отсчета. В поле ЧД температура также уменьшается с увеличением расстояния от ЧД. Однако она стремится к постоянному значению $1 / 8 \pi G M$ для бесконечно далекого наблюдателя. Температура остается конечной (и близкой к этой постоянной) в бесконечном интервале расстояний от ЧД. Следовательно, в случае ЧД сушествуют широкие коридоры, полностью лежашие в области, далекой от начала отсчета, и тем не менее содержашие траектории 
термических частищ.

Рассмотрим измерение, соответствуюшее коридору рис. 7а, имеющему ширину больше, чем типичная длина волны термических частиц $\lambda=1 / k T$. Альтернативные результаты измерения описываются в этом случае числом и положением ворот, которые также достаточно широки. Число ворот (деленное на 2) определяет число термических частищ, наблюденных при данном результате измерения. Вследствие того что как коридор, так и ворота являются широкими, все наблюденные частицы реальны (а не возникают в результате слишком сильной локализации во время измерения). Однако никакая частица при этом не поглощается и не испускается черной дырой, так что масса ЧД не может измениться за счет процессов такого типа. Этого, разумеется, и следовало ожидать в случае вечной ЧД.

Естественно возникает вопрос: если наблюдаемая термическая частица не отличается от реальной, то должна ли она нести энергию и давать вклад в общую массу ЧД и ее окружения, как эта масса представляется далекому наблюдателю? Ответ на этот вопрос является утвердительным. Каждая термическая частица дает вклад в общую энергию. Однако сумма всех таких вкладов равна нулю. Это связано со специфическими свойствами времени далекого наблюдателя. Поверхность постоянного времени для такого наблюдателя представляется на диаграмме Крускала рис. 7 прямой линией, проходящей через начало отсчета. Если правый конец такой линии движется вверх (положительное направление времени), то ее левый конец движется вниз.

Из-за того что пути термических частиц являются частями одной петли, такие частицы разделяются на пары, каждая из которых состоит из частицы и античастицы: частища - в правом квадранте (область, причинно связанная с наблюдателем), а античастица - в левом. Относительно времени наблюдателя каждая частица такой пары распространяется в положительном направлении времени (в будушее), в то время как соответствующая античастица распространяется в отрицательном направлении времени (в прошлое). Следовательно, если частица имеет положительную энергию, то античастица - отрицательную. Вследствие полной симметрии множества всех путей эти энергии компенсируют друг друга, так что полный вклад всех пар в массу, воспринимаемую удаленным наблюдателем, равен нулю.

Если, однако, одна из частиц поглошается наблюдателем (коридор рис. 7б), симметрия нарушается в момент поглощения (по времени этого наблюдателя). Начиная с этого момента число античастиц на единицу больше, чем число частиц. Поглошение термической частицы сопровождается другим процессом: одна из античастищ становится реальной и падает на будушую сингулярность ЧД. Отрищательная энергия этой античастицы дает теперь вклад в общую массу, которую наблюдатель (поглотивший частицу) интерпретирует как массу ЧД. Измеряя гравитационное поле, этот наблюдатель увидит, что оно соответствует теперь меньшей массе. Он интерпретирует этот факт как уменьшение массы ЧД, вызванное поглощением термической частишы наблюдателем и 
падением соответствующей античастицы, имеющей отрицательную массу, на будушую сингулярность ${ }^{6}$.

Выводы для вечной (шварцшильдовской) ЧД:

А. Частищы, составляющие "термическую атмосферу" далекого наблюдателя, невозможсно отличить от реальных частич, но они не меняют массу ЧД, если только не поглощаются. Вместе со своими партнерами-античастицами они образуют петлю в пространстве-времени, и их әнергии (относительно времени далекого наблюдателя) компенсируют друг друга.

Б. Поглощение частищы далеким наблюдателем сопровождается падением античастицы на сингулярность, что приводит к изменению массы ЧД, как она определяется далеким наблюдателем (например, по величине гравитационного поля). Вместо әтого поглощенная частица может испускаться из прошлой сингулярности.

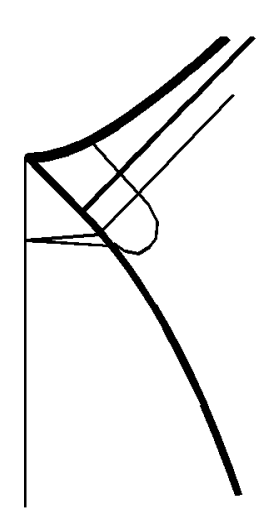

a

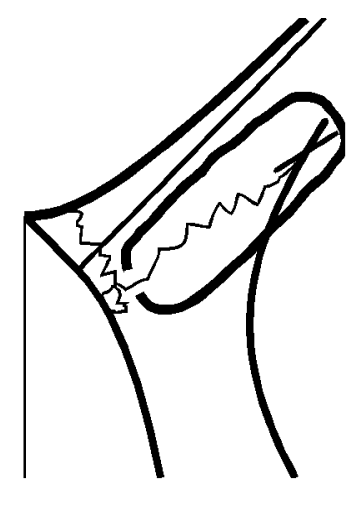

б

Рис. 8. ЧД, формирующаяся в процессе коллапса: а - превращение виртуальной пары в реальную в точке, в которой начинается горизонт; б - поглощение наблюдателем термической частицы и измерение ее траектории начиная от точки, где она рождается.

5.2. Черная дыра, формирующаяся при коллапсе. Рассмотрим теперь ЧД, формируюшуюся при коллапсе (рис. 8). По сравнению с вечной ЧД такая формируюшаяся ЧД обладает одной сушественно новой чертой. В пространственно-временной точке, совпадаюшей с началом горизонта событий, виртуальная пара может быть "разорвана" и при этом образуются реальная частица с энергией порядка $k T=1 / 8 \pi G M$, улетаюшая на бесконечность, и реальная античастица, падаюшая в ЧД (рис. 8а). Механизм этого состоит в следуюшем [18]. Частица, имеющая энергию порядка $k T$ и входя-

\footnotetext{
${ }^{6)}$ Вместо будущей сингулярности свободный конец разорванной петли может начинаться на прошлой сингулярности. Тогда поглощенная частица испускается черной дырой.
} 
щая в состав виртуальной пары, может пройти сквозь коллапсирующее тело (которое на этой последней стадии коллапса имеет размер порядка $8 \pi G M$, т.е. порядка длины волны $\lambda=1 / k T$ этой частицы), выйти из него с другой стороны и улететь на бесконечность. В то же время античастица, входящая в эту виртуальную пару, может миновать коллапсируюшее тело и упасть в ЧД.

Как доказано в работе [18], спектр энергий частиц, улетающих на бесконечность посредством такого механизма, является термическим с температурой $k T=1 / 8 \pi G M$. Это чрезвычайно похоже на то, что имеет место вокруг вечной ЧД (хотя механизмы явления в этих двух случаях различны). Однако в случае ЧД, образующейся при коллапсе, “термическая атмосфера" состоит из реальных, а не виртуальных частиц. Рождение каждой из этих частиц сопровождается рождением античастицы, несушей отрицательную энергию в ЧД. Масса ЧД в результате такого процесса уменьшается. Любая из термических частиц, возникающих таким образом, может быть поглощена далеким наблюдателем. Происхож дение частищы, которая наблюдается (или поглошается), может быть в принципе прослежено вплоть до момента ее возникновения в области, где формируется горизонт (рис. 8б).

Выводы для ЧД, возникаюшей в процессе коллапса:

А. Вблизи пространственно-временной точки, в которой начинается горизонт событий, виртуальная пара может превратиться в реальную, при этом античастица падает в ЧД, уменьшая ее массу, а частица ускользает на бесконечность вдоль горизонта.

Б. Реальные частицы, ускользающие на бесконечность, могут наблюдаться (и поглощаться) далеким наблюдателем. Однако независимо от их поглощения эти частицы уносят массу Ч,Д.

В. Траектория поглощенной (или наблюденной) частицы может быть в принципе прослежсена начиная от точки вблизи начала горизонта, где частица рожсдается.

\section{6. ЗАКЛЮЧИТЕЛЬНЫЕ ЗАМЕЧАНИЯ}

Техника релятивистских ОИП и квантовых коридоров была здесь описана лишш в обших чертах. Некоторые важные процедуры, характерные для этой техники, не были должным образом обсуждены, например процедура суммирования по всем альтернативным результатам измерения. Кроме того, ни один ОИП не был реально вычислен. Тем не менее оценка ширины квантовых коридоров в различных физических ситуациях привела нас к некоторым новым заключениям или по крайней мере сделала более ясной некоторые точки зрения на эффекты Унру и Хокинга.

Главный из сделанных выводов состоит в тонком различии между следующими случаями: а) эффекта Унру, б) вечной ЧД и в) ЧД, возникаюшей в процессе коллапса. Природа “термической атмосферы” наблюдателя в этих трех случаях различна. Она 
состоит из виртуальных частищ в случае "а" и из реальных частиц в случае "в". В промежуточном случае "б" термические частицы могут наблюдаться в достаточно широкой области, так что они ничем не отличаются от реальных. Если такая частица поглошается, масса ЧД уменьшается на величину энергии поглошенной частицы. Однако пока термические частицы не поглощены, они не уносят массу ЧД, так что эта масса остается постоянной ${ }^{7}$. Фактически термические частицы приобретают все больше черт реальных частиц, если двигаться по цепочке "а" $\rightarrow$ "б" $\rightarrow$ "в".

Различие между вечной ЧД и коллапсируюшим телом может иметь последствия для астрофизических наблюдений. Если наблюдается некоторая ЧД, не обязательно ожидать, что она в конце концов испарится. Случится это или нет, зависит от предыстории Ч.Д. Если ЧД была сформирована в процессе коллапса, она в конце концов испарится, но если она существовала во все времена в прошлом, то она будет существовать бесконечно также и в будущем. Такая ЧД является действительно "вечной". По крайней мере это верно в случае, если окружение ЧД не слишком плотно, т.к. в противном случае поглощение этим окружением частиц из “термической атмосферы” приведет к падению соответствуюших античастиц в ЧД и к уменьшению наблюдаемой массы ЧД.

Эта работа поддержана Российским фондом фундаментальных исследований, грант 95-01-00111a.

\section{Список литературы}

[1] K.-E. Hellwig, K. Kraus. Phys. Rev. D. 1970. V. 1. P. 566

[2] Y. Aharonov, D. Z. Albert. Phys. Rev. D. 1981. V. 24. P. 359.

[3] Y. Aharonov, D. Z. Albert. Phys. Rev. D. 1984. V. 29. P. 228; S. Malin. Phys. Rev. D. 1982. V. 26. P. 1330; E. J. Squires. Phys. Lett. A. 1990. V. 145. P. 297; S. N. Mosley. Phys. Lett. A. 1994. V. 182. P. 1; J. Finkelstein. Found. Phys. Lett. 1992. V. 5. P. 383.

[4] J. Finkelstein. Phys. Lett. A. 1994. V. 188. P. 117.

[5] M. B. Mensky. Phys. Rev. D. 1979. V. 20. Р. 384; М. Б. Менский. ЖЭТФ. 1979. Т. 77. C. 1326 .

[6] M. B. Mensky. Continuous Quantum Measurements and Path Integrals. Bristol, Philadelphia: IOP Publishing, 1993.

[7] M. B. Mensky, H. von Borzeszkowski. Phys. Lett. A. 1995. V. 208. P. 269.

[8] R. P. Feynman. Rev. Mod. Phys. 1948. V. 20. P. 367.

[9] J. Audretsch, M. B. Mensky. Phys. Rev. A. 1997. V. 56. P. 44.

[10] Y. Aharonov, M. Vardi. Phys. Rev. D. 1980. V. 21. P. 2235; Ф. Я. Халили. Вестн. Моск. ун-та. Cер. 3, физ., астр. 1981. T. 22. С. 37; A. Barchielli, L. Lanz, G. M. Prosperi. Nuovo Cimento B. 1982. V. 72. P. 79; C. M. Caves. Phys. Rev. D. 1986. V. 33. P. 1643; 1987. V. 35. P. 1815 .

[11] Howard Carmichael. An Open Systems Approach to Quantum Optics. Berlin, Heidelberg: Springer-Verlag, 1993.

\footnotetext{
7) Различие между вечной ЧД и ЧД, формирующейся в коллапсе, обсуждалось в работах [21]. Вывод из этого обсуждения состоял в том, что вакуум должен быть стабильным в поле вечной Чд, но не в случае коллапсируюшего тела.
} 
[12] William G. Unruh, Robert M. Wald. Phys. Rev. D. 1984. V. 29. P. 1047.

[13] E. C. Stueckelberg. Helv. Phys. Acta. 1941. V. 14. P. 322; 1942. V. 15. P. 23.

[14] М. Б. Менский. ТМФ. 1974. Т. 18. С. 190; M. B. Mensky. Lett. Math. Phys. 1978. V. 2. P. 175; Helv. Phys. Acta. 1996. V. 69. P. 301.

[15] W. G. Unruh. Phys. Rev. D. 1976. V. 14. P. 870.

[16] W. Troost, H. Van Dam. Phys. Lett. B. 1977. V. 71. P. 149; Nucl. Phys. B. 1979. V. 152. P. 442.

[17] N. D. Birrell, P. C. W. Davies. Quantum Fields in Curved Space. Cambridge: Cambridge University Press, 1982.

[18] S. Hawking. Nature. 1974. V. 248. P. 30; Commun. Math. Phys. 1975. V. 43. P. 199.

[19] W. G. Unruh. Phys. Rev. D. 1992. V. 46. P. 3271.

[20] Robert M. Wald. Quantum field theory in curved spacetime and black hole thermodynamics. Chicago: University of Chicago Press, 1994.

[21] М. Б. Менский. Письма в ЖЭЭТФ. 1976. Т. 24. С. 561; M. В. Mensky. On stability of the vacuum for a class of gravitational fields including black hole field. In: Proceed. 18th Intern. Conf. High Energy Phys. (Tbilisi, USSR, 1976). V. II. Dubna: JINR, 1977. P. 30.

Поступила в редакцию $30 . X I I .1997$ г. 